Shaw Jonathan (Orcid ID: 0000-0002-6187-2203)

Blonde Lawrence (Orcid ID: 0000-0003-0492-6698)

Paul Sanjoy (Orcid ID: 0000-0003-0848-7194)

Title: Long-term sustainability of glycaemic achievements with second-line anti-diabetic therapies in patients with type 2 diabetes: A real-world study.

Short Title: Glycaemic achievements with second-line anti-diabetic agents.

Olga Montvida, $\mathrm{MSc}^{1,2}$, Jonathan $\mathrm{Shaw}^{3}$, Lawrence Blonde, $\mathrm{MD}^{4}$, Sanjoy K Paul, $\mathrm{PhD}^{1,5}$

${ }^{1}$ Statistics Unit, QIMR Berghofer Medical Research Institute, Brisbane, Australia

${ }^{2}$ School of Biomedical Sciences, Faculty of Health, Queensland University of Technology, Brisbane, Australia

${ }^{3}$ Baker Heart and Diabetes Institute, Melbourne, Victoria, Australia

${ }^{4}$ Ochsner Diabetes Clinical Research Unit, Frank Riddick Diabetes Institute, Department of Endocrinology, Ochsner Medical Center, New Orleans, LA

${ }^{5}$ Melbourne EpiCentre, University of Melbourne and Melbourne Health, Melbourne, Australia

Corresponding Author and person to whom reprint requests should be addressed:

Professor Sanjoy Ketan Paul

The Royal Melbourne Hospital - City Campus 17 East, Main Building

Grattan Street, Parkville Victoria 3050

Email: Sanjoy.Paul@unimelb.edu.au

Phone: +61393428433

Fax: +61393428780

Keywords: glycaemic control, anti-diabetic drug, therapeutic choice,

Word Count, Abstract: 250

Word Count, Main Body: 3665

Number of Tables: 2

This is the author manuscript accepted for publication and has undergone full peer review but has not been through the copyediting, typesetting, pagination and proofreading process, which may lead to differences between this version and the Version of Record. Please cite this article as doi: 10.1111/dom.13288

This article is protected by copyright. All rights reserved. 
Number of Figures: 2

Supplemental Figures: 1

Number of References: 31

This article is protected by copyright. All rights reserved. 


\section{ABSTRACT}

Aims: To inform patients and their carers about the probability of reducing HbAlc to clinically desirable levels and sustainability of such control over 2 years with major secondline anti-diabetic therapies under individual risk scenario, with and without third-line intensification.

Materials and Methods: From US Centricity Electronic Medical Records, 163,081 patients with type 2 diabetes aged 18-80 years, who initiated metformin; intensified with DPP-4 inhibitor (DPP-4i), GLP-1 receptor agonist (GLP-1RA), sulfonylurea, insulin, or thiazolidinedione; and continued second-line e 6 month, were selected. Treatment groups were balanced on baseline characteristics and glycaemic achievements were estimated with logistic regression.

Results: With HbA1c 7.5-7.9\% at second-line initiation, the adjusted probabilities to achieve HbAlc $<7 \%$ at 6 month were 32/38/39/26/38\% in the sulfonylurea/ DPP-4i/ GLP-1RA/ insulin/ thiazolidinedione groups. With baseline HbA1c of $8-9 \%$, the probabilities of reducing $\mathrm{HbAlc}<7.5 \%$ were $38 / 44 / 40 / 34 / 42 \%$ respectively. In these baseline HbA1c categories, the adjusted probabilities of sustaining $\mathrm{HbAlc}$ achievements over 2 years were higher in the GLP-1RA and thiazolidinedione groups, compared to sulfonylurea and insulin $(\mathrm{p}<0.01)$. With baseline HbAlc of $9.1-12 \%, 38 \%$ achieved $\mathrm{HbA} 1 \mathrm{c}<7.5 \%$ at 6 months. The adjusted probability of sustaining this control over 2 years was higher in the incretins and thiazolidinedione groups (range: 62-75\%), while insulin and sulfonylurea offered lower chances of sustainable control (range: $54-56 \%$ ). 
Conclusions: Patients treated with second-line incretins and thiazolidinedione had significantly higher probability of achieving and sustaining glycaemic control over two years without further intensification, compared to those treated with sulfonylurea or insulin.

\section{INTRODUCTION}

Metformin (MET) is recommended as a first-line pharmacological treatment for patients with type 2 diabetes. However, most patients eventually require therapy intensification with multiple anti-diabetic drugs (ADDs) for glycaemic control ${ }^{1-3}$. For second-line intensification, the American Diabetes Association recommends: sulfonylurea (SU), thiazolidinedione (TZD), DPP-4 inhibitor (DPP-4i), SGLT-2 inhibitor, GLP-1 receptor agonist (GLP-1RA), or insulin (INS); other drugs are recommended under specific conditions ${ }^{1}$. The temporal patterns of the changes in the second-line ADD choices over the last decade in the US have been recently explored by Montvida et al (2017) ${ }^{4}$.

While clinicians' and patients' decisions on an add-on agent have become more complicated 5, 6 , few studies directly compare glycaemic effectiveness of second-line therapies ${ }^{7-11}$. A recent network meta-analysis reported similar glycaemic achievements by all second-line ADDs, when added to $\mathrm{MET}^{9}$. Analogous results were discussed in two observational studies using electronic medical records (EMRs) ${ }^{7,8}$. In 7,009 patients from Germany, Rathman and colleagues (2016) reported unadjusted mean $\mathrm{HbAlc}$ reduction of $0.7-1.1 \%$ at 6 months of treatment by major second-line ADDs including insulin ${ }^{7}$. The Danish study on 4,734 patients by Thomsen and colleagues (2015) reported a median reduction of $0.8-1.3 \%$ at 12 months 
for non-insulin drugs (from baseline $\mathrm{HbA1c}$ of $7.6-8 \%$, and $2.4 \%$ for insulin treated patients $(\text { from } 9.6 \%)^{8}$.

Given the increasing complexity and challenges in multiple risk factor management in patients with type 2 diabetes, and the availability of a number of new and older classes of ADDs, a population-level assessment of the likelihood of short- and long-term glycaemic achievements and their sustainability, by different second-line ADDs, would be of great value. With evaluation of a reasonably large number of patients from primary and ambulatory care systems, probabilistic estimates of sustainable glycaemic achievements by different second-line ADDs in patients under different risk paradigms would empower clinicians and their patients to make more informed therapeutic choices. To the best of our knowledge, no study has evaluated early glycaemic control, and its sustainability across different levels of HbAlc at post-MET second-line ADD intensification.

The newer classes of ADDs including GLP-1 RA and SGLT-2 inhibitors, have potential extra glycaemic benefits such as weight and blood pressure reductions along with possible association with reduced risk of cardiovascular diseases. However, these therapies are costlier in comparison to the older ADDs. If newer drugs have longer lasting benefits on glycaemic control than older ADDs, then over time, lower rate of third-line therapy intensification across the whole population is expected, as the use of newer drugs increases. In this context, evaluation of whether second-line therapy intensification with newer drug classes has been helpful in reducing the need for third-line therapy intensification over time, is of great interest. However, there is a paucity of population-level studies on this question. A modelling study by Zhang and colleagues (2014) reported marginally shorter time to insulin in patients 
treated with incretin-based therapies (GLP-1RA and DPP-4i) compared to those treated with $\mathrm{SU}^{12}$. However, we are not aware of any population-level study that evaluated the possible delay in the need for third-line therapy intensification in patients who choose incretin-based therapies as second-line.

With heterogeneous $\mathrm{HbA} 1 \mathrm{c}$ levels at second-line initiation, the main aims of this study were to inform clinicians and patients on (1) the likelihood of reducing HbAlc to a clinically desirable level over 6, 12 and 24 months of treatment with major second-line ADDs when added to MET, (2) the probability of sustaining early glycaemic control over 24-months of therapy continuation with and without the need for third-line ADD addition, and (3) whether the availability of newer ADDs have reduced the need for intensification with third-line therapy at the population-level over time.

\section{MATERIALS AND METHODS}

\section{Data Source}

The Centricity Electronic Medical Record (CEMR) of USA was used in this study, it represents more than 35,000 solo practitioners, community clinics, academic medical centres, and large integrated delivery networks across all US states. Patients in the database are generally representative of the USA population, with a diabetes prevalence $(7.1 \%$ identified by diagnostic codes) that is similar to National Diabetes Statistics $(6.7 \%$ diagnosed diabetes in 2014) $)^{13}$. CEMR has been extensively used for academic research worldwide ${ }^{14-16}$. 
For more than 34 million individuals, longitudinal EMRs were available from 1995 until April 2016, with comprehensive patient-level information on demographics, anthropometric, clinical and laboratory variables. Medication data include brand names and doses for individual medications prescribed, along with start/stop dates and specific fields to track treatment alterations. This dataset also contains patient reported medications, including prescriptions received outside the EMR network and over-the-counter medications.

\section{Study Design}

For each identified patient with type 2 diabetes and non-missing age and sex. To obtain data on the first-, second-, and third-line ADDs for each patient, the following drug classes were arranged chronologically according to the initial prescription dates: MET, SU, TZD, AGI, amylin, dopamine receptor agonist, meglitinide, DPP-4i, GLP-1RA, SGLT-2 inhibitor, and INS. Same day initiations (including combination therapies) were prioritized in the order as listed above, from highest to lowest. A robust methodology for extraction and assessment of longitudinal patient-level medication data from the CEMR database has been recently described by the authors ${ }^{17}$.

Study cohort included patients with: (1) age at diagnosis $\geq 18$ and $<80$ years, (2) diagnosis date strictly after first registered activity in the CEMR database, (3) diagnosis date on or after January 1, 2005, (4) initiated anti-diabetic therapy with MET, (5) initiated second-line ADD with SU, TZD, DPP-4i, GLP-1RA or INS, (6) available HbA1c measure at second-line ADD initiation (baseline), and (7) second-line therapy duration $\geq 6$ months. Additional restrictions 
on the duration of second-line therapy were applied: $\geq 12$ months (sub-cohort 1 ) and $\geq 24$ months (sub-cohort 2).

Baseline body weight, BMI, systolic/diastolic blood pressure, and lipids were calculated as the average of available measurements within the 3 months before and 3 months after initiation of therapy. HbA1c measures at baseline, $6,12,18$, and 24 months were obtained as the nearest measure within 3 months either side of the time point. With the condition of at least two non-missing follow-up data over 24 months, the missing data were imputed using a Markov Chain Monte Carlo method adjusting for age, diabetes duration and usage of concomitant $\mathrm{ADDs}^{18}$. Next, the following baseline HbA1c categories were created: (1) 7.0$7.9 \%$ (2) $8.0-9.0 \%$ (3) $9.1-12.0 \%$, and (4) $>12 \%$.

The presence of comorbidities prior to baseline was assessed by relevant disease identification codes. The Charlson Comorbidity Index (CCI) was calculated following the algorithm described by Quan and colleagues ${ }^{19}$. Cardiovascular disease (CVD) was defined as ischaemic heart disease, peripheral vascular disease, heart failure, or stroke. Cancer was defined as any malignancy except malignant neoplasm of skin.

\section{Statistical Methods}

Baseline characteristics were summarised as number (\%), mean (SD) or median (first quartile, third quartile) as appropriate. Patterns of intensification with third ADD were summarised by second-line ADDs in the study cohort, sub-cohort 1, and sub-cohort 2 . Among patients with $\geq 2$ years of follow-up in the study cohort, proportions $(95 \% \mathrm{CI})$ of 
those who initiated third ADD within 2 years of baseline were calculated by year of secondline initiation.

Propensity scores for multiple treatment levels ${ }^{20}$ were calculated within each $\mathrm{HbA}$ c category to account for heterogeneous baseline characteristics among second-line ADD groups. Inverse probability of these exposure weights (IPTW) ${ }^{21,22}$ were used to balance second-line treatment groups on age, sex, baseline $\mathrm{HbAlc}$, and baseline CCI. In patients without history of CVD, chronic kidney disease (CKD), or cancer at baseline, probabilities (95\% CIs) of achieving glycaemic control (HbA1c below 7 or $7.5 \%)$ at 6,12 , and 24 months post secondline initiation were estimated in the study cohort, sub-cohort 1, and sub-cohort 2 respectively. Three outcomes were assessed with multinomial logistic regression: (1) no glycaemic achievement at corresponding time point, (2) glycaemic achievement with a third ADD addition within the analysis time window, and (3) glycaemic achievement without a third ADD addition within the analysis time window. Analyses were conducted by balancing the data as described above, with additional covariate adjustments on age, sex, and time from MET to second-line, separately for the HbAlc categories of 7.5-7.9\%, 8.0-9.0\% and 9.1$12.0 \%$.

In patients with baseline $\mathrm{HbAlc} 7.5-7.9 \%$, who achieved $\mathrm{HbA} 1 \mathrm{c}$ target of $7 \%$ at 6 months without third ADD addition, the probabilities to sustain HbA1c control over 24 months were estimated with balancing and adjustments as described above. Similarly, in patients with baseline $\mathrm{HbA} 1 \mathrm{c}$ of $8-9 \%$ who achieved $\mathrm{HbA1c}$ below 7.5\% at 6 months without third ADD addition, the adjusted probabilities to sustain HbA1c control over 24 months were estimated. Finally, in patients with baseline HbA1c of $9.1-12 \%$, who achieved HbAlc below $7.5 \%$ at 6 
months with or without third-line intensification, the adjusted probabilities to sustain $\mathrm{HbA1c}$ control (irrespective of third ADD status) over 24 months were estimated. The assessment of achieving $\mathrm{HbA} 1 \mathrm{c}<7 \%$ in this category was considered clinically unrealistic.

Sensitivity analyses included an intention-to-treat evaluation and separate assessment in patients with comorbidities at baseline.

\section{RESULTS}

From 2,624,954 identified patients with type 2 diabetes, 195,720 initiated second-line ADD post MET and had available HbA1c measure (Supplementary Figure 1). Of them, 85/ 79/77/ $83 / 83 \%$ in the SU/ DPP-4i/ GLP-1RA/ INS/ TZD groups continued therapy for at least 6 months respectively. The study cohort included 90,572 / 29,308/ 6,696/ 21,827/ 14,678 patients in the SU/ DPP-4i/ GLP-1RA/ INS/ TZD groups (Table 1). On average, the progression to a second ADD occurred 9 months post MET initiation. Available follow-up years from baseline were 4.0/ 3.2/ 3.7/ 3.5/ 5.6 years in the SU/ DPP-4i/ GLP-1RA/ INS/ TZD groups, and $84 \%$ of patients continued therapy for at least 1 year. The distributions of

age, sex, BMI and comorbidities at baseline were significantly different between the secondline ADDs (Table 1).

The distribution of $\mathrm{HbAlc}$ categories at baseline was heterogeneous among the treatment groups (Table 1). With a mean (SD) cohort HbA1c level of $8.4(1.9) \%$ at second-line therapy initiation, the proportions of patients with baseline HbA1c below 8.0\% was 52/ 58/ 67/ 36/ $66 \%$ in the SU/ DPP-4i/ GLP-1RA/ INS/ TZD groups respectively.

\section{Treatment intensification with a third drug}


Overall, $52 \%$ in the cohort had a third ADD prescribed (either in addition or as a switch from second ADD) during available follow-up. On average, the progression to a third ADD occurred at 15 months post second-line initiation (Table 2). Of those who initiated a third drug, $88 \%$ added it on top of dual therapy (ranging from $70 \%$ in the INS group to $94 \%$ in GLP-1RA group), while only $12 \%$ ceased the second ADD and switched to a third agent.

By study design, patients who switched to a third agent within 6/ 12/ 24 months were not included in the study cohort/ sub-cohort $1 /$ sub-cohort 2 respectively. During 6 months of therapy post baseline, $27 / 21 / 26 / 12 / 29 \%$ patients added third-line therapy in the SU/ DPP4i/ GLP-1RA/ INS/ TZD groups respectively (Table 2). INS was the most popular third ADD, followed by DPP-4i. Of those who added a third drug, INS was chosen by $26 / 36 / 69$ / $32 \%$ of patients in the SU/ DPP-4i/ GLP-1RA/ TZD groups respectively (Table 2). Among those who continued the second-line therapy for 12 months (sub-cohort 1) and for 24 months (sub-cohort 2), 30\% and 39\% added a third-line therapy respectively.

\section{Temporal pattern of initiating third-line ADD}

Irrespective of the class of second-line ADD, the proportions of patients who initiated a third ADD within 2 years of baseline are shown in the Figure 1A ("All”) by calendar year of second-line initiation. Figure 1 also depicts those who intensified with a third ADD excluding TZD as second-line group (“All without TZD”) as large portion of patients were ceasing TZD treatment due to cardiovascular safety concerns ${ }^{23,24,25}$ and not necessarily due to efficacy issue. We also provide a line excluding those who had TZD or INS as second-line ("All without TZD \& INS") to explore the possible change in intensification rate with non-insulin 
ADDs over time, accounting for decreasing popularity of TZD. . Figures B and C focus on those who had baseline $\mathrm{HbA1c}$ of $8-9 \%$ and $9.1-12 \%$ respectively. Figure 1 shows that from 2007-2014 the proportion of patients initiating a third ADD, within two years of adding the second ADD, fell. However, this decline started to reverse in 2014, especially among those whose $\mathrm{HbA1}$ c was $9.1-12 \%$ at initiation of the second ADD.

\section{Glycaemic Achievements and Sustainability}

At 6 months, the mean unadjusted $\mathrm{HbAlc}$ reductions were $0.8 / 0.8 / 0.7 / 1.0 / 0.8$ percentage points in the SU/ DPP-4i/ GLP-1RA/ INS/ TZD groups respectively. Mean adjusted reductions at 6 months were $0.8 / 1.0 / 1.1 / 0.7 / 1.0$ percentage points in the respective treatment groups (significant for all groups, $\mathrm{p}<0.01$ ).

\section{Baseline HbA1c: 7.5-7.9\%}

Among patients with HbA1c 7.5-7.9\% at baseline, 44/ 47/ 57/ 31/ $57 \%$ of patients in the SU/ DPP-4i/ GLP-1RA/ INS/ TZD groups achieved HbA1c below 7\% at 6 months without thirdline addition. The corresponding adjusted probabilities were $32 / 38 / 39 / 26 / 38 \%$ in the second-line treatment groups (Figure $2 \mathrm{~A}, \mathrm{p}<0.01$ for all groups). However, the probabilities of reducing $\mathrm{HbA1c}$ below 7\% target without third ADD intensification declined by $5 / 5 / 6 / 2 /$ $1 \%$ at 12 months and by $9 / 8 / 15 / 5 / 7 \%$ at 24 months in the SU/ DPP-4i/ GLP-1RA/ INS/ TZD groups respectively.

Among those who reduced HbA1c below 7\% without a third ADD at 6 months, $68 \%$ and $58 \%$ of patients sustained glycaemic achievement at 12 and 24 months respectively. The probability of sustaining this glycaemic achievement was higher and similar in the GLP-1RA 
and TZD groups at 12 months (range of 95\% CI of probability: 76, 79\%), compared to other second-line therapy options (Figure 2B). While the probability of sustaining this glycaemic control declined significantly by 24 months, GLP-1RA, DPP-4i and TZD provided significantly higher chances of sustainability (range of 95\% CI of probability: $53,58 \%$ ) compared to patients treated with INS or SU (range of $95 \%$ CI of probability: $46,50 \%$ ).

\section{Baseline HbA1c: 8.0-9.0\%}

Among patients with baseline HbA1c of $8-9 \%, 55 / 58 / 66 / 41 / 67 \%$ of patients in the SU/ DPP-4i/ GLP-1RA/ INS/ TZD groups achieved HbA1c below 7.5\% at 6 months without third-line ADD addition, and the corresponding adjusted probabilities were 38/44/ 40/ 34/ $42 \%$ respectively (Figure $2 \mathrm{C}$ ). The probabilities of this glycaemic achievement declined significantly by at least 5\% across all treatment groups at 12 months, and by at least $8 \%$ at 24 months.

Among those who reduced HbA1c below 7.5\% without third ADD at 6 months, $76 / 67 \%$ sustained glycaemic achievement at 12/ 24 months without requiring third-line intensification. The probability of sustaining this glycaemic achievement was significantly higher in the GLP-1RA and TZD groups at 12 months (range of 95\% CI of probability: 76, $79 \%$ ), compared to other second-line ADDs (Figure 2D, $\mathrm{p}<0.01$ ). While the probability of sustaining this glycaemic control declined significantly by 24 months of therapy across all groups, patients treated with INS had the lowest probability of sustaining the glycaemic control.

\section{Baseline HbA1c: 9.1-12.0\%}


In the patients with 9.1-12\% baseline HbA1c, 29/36/ 45\% added third ADD within 6/ 12/ 24 months of baseline respectively. Irrespective of third ADD status, 37/ 45/38/21/43\% of patients in the SU/ DPP-4i/ GLP-1RA/ INS/ TZD groups achieved HbA1c below 7.5\% at 6 months, with corresponding probabilities of 36/45/38/33/43\% (Figure 2E). The probability to reduce $\mathrm{HbA} 1 \mathrm{c}$ below $7.5 \%$ at 24 months reduced by $4 \%$ for INS users, did not changed in SU and DPP-4i groups, and increased by 8 and $9 \%$ in the second-line GLP-1RA and TZD groups (all $\mathrm{p}<0.01$ ). Among those who reduced HbA1c below $7.5 \%$ at 6 months, $72 / 58 \%$ sustained glycaemic achievement at $12 / 24$ months irrespective of third-line intensification status. The probability of sustaining glycaemic control below $7.5 \%$ over 12 - and 24 -months of treatment was significantly higher in the incretins and TZD groups, while INS and SU offered lower chances of sustainable control. (Figure 2F).

\section{Baseline HbA1c $>\mathbf{1 2 . 0} \%$}

In patients with baseline $\mathrm{HbA} 1 \mathrm{c}>12 \%$, probabilities to reduce $\mathrm{HbA} 1 \mathrm{c}$ for at least $2 \%$ were increasing over time: $82 \%$ at 2 years of INS therapy, and approximately $90 \%$ for other second-line choices. The probabilities to reduce $\mathrm{HbA} 1 \mathrm{c}$ for at least $1.5 \%$ in this baseline HbAlc group were not significantly different among the ADD groups over 2 years (results not shown).An intention to treat approach revealed similar results to the main analyses. Patients with CVD, CKD or cancer at baseline had marginally higher probabilities of glycaemic achievements in all treatment groups, compared to those without comorbidities (results not shown).

\section{DISCUSSION}


The novelty of this pharmaco-epidemiological study with real-world population-level data is the evaluation of short- and long-term glycaemic control with post-metformin major secondline ADDs, and the comparison of the sustainability of such glycaemic goals over 24 months of continuous treatment. Among patients with $\mathrm{HbAlc} 7.5-7.9 \%$ at second-line ADD initiation, the probability of achieving the $\mathrm{HbA} 1 \mathrm{c}$ below $7 \%$ without adding third-line ADD at 6 and 12 months were significantly higher in the incretins and TZD groups, compared to the INS and SU groups. Treatment with incretins or TZD also offered a significantly higher probability of sustaining this glycaemic achievement over 24 months of treatment without the need for further therapy intensification. Among those who initiated second-line ADD at 8-9\% HbA1c level, DPP-4i and TZD offered significantly higher and similar chances of reducing HbA1c below $7.5 \%$ over 24 months of therapy continuation without adding third ADD, compared to other second-line groups. GLP-1RA and TZD offered the highest chances of sustaining this control over 24 months, while treatment with SU, INS and DPP-4i provided significantly lower sustainability chances.

In this real-world study, we have observed similar performance of DPP-4i and GLP-1RA in terms of the probability of reducing $\mathrm{HbA} 1 \mathrm{c}$ to a clinically desirable glycaemic target over 24 months of therapy, when added to metformin. In terms of sustaining the glycaemic achievements over 12 months, GLP-1RA appears to offer higher chances among patients with $\mathrm{HbA} 1 \mathrm{c}$ below $9 \%$ at second-line initiation ( $76-79 \%$ probability), compared to DPP-4i ( $\sim 68-73 \%$ probability). However, this difference disappears at 24 months of therapy. While SU as second-line therapy offers higher probability of achieving desirable glycaemic control across all HbA1c categories $(<12 \%)$ compared to INS over two years, the probability of 
sustaining the early glycaemic achievement appears to be similar between these two therapy options. We have seen that across all HbA1c categories, treatment with second-line TZD provided better or similar glycaemic achievements and sustainability, compared to other therapy options. This result supports study of Mamza and colleagues (2016), reporting that treatment with post-metformin TZD provides most durable glycaemic response compared to second-line SU and DPP- $4 \mathrm{i}^{26}$. Recent results of the TOSCA.IT trial, providing cardiovascular safety reassurance with pioglitazone, taken in conjunction with our results may increase the popularity of TZDs as a therapeutic option ${ }^{27}$.

Compared to sulfonylurea add-on to metformin, Thomsen and colleagues reported higher likelihood of achieving HbA1c below 7\% at 6 months for second-line GLP-1RA (95\% CI of RR: 1.01-1.19) users, and lower likelihoods for DPP-4i (95\% CI of RR: 0.89-0.99) and INS (95\% CI of RR: $0.77-0.99)$ users $^{8}$. Our results are closer to the study conducted by Rathman and colleagues, who reported odds ratios (with SU as reference) of achieving HbA1c below $7 \%$ of $1.2 / 1.4 / 1.7 / 0.7$ for second-line DPP-4i/ GLP-1RA/ TZD/ INS respectively.

Our findings are also in line with a study that using data from the National Health and Nutrition Examination Survey reported that only half of patients achieve HbAlc below $7.0 \%{ }^{28}$. Furthermore, in patients with $\mathrm{HbAlc}<9 \%$ at second-line initiation, we have observed that only $30 \%$ maintain glycaemic control after 2 years of continuous treatment without further intensification with a third ADD.

Comparatively poor performance of insulin as a second-line agent may be surprising, as RCT data show insulin to achieve at least as much HbAlc lowering as other agents. The possible 
reason for these findings is that insulin is often chosen when there are multiple comorbidities, and in such patients, the HbAlc target may be higher, and many other potential third-line ADDs may be contra-indicated. Second, the insulin dose may be inadequately titrated, the reasons may include adverse-effects such as hypoglycaemia and weight gain, as well as inadequate healthcare professional support for the regular titration of insulin doses. More work needs to be done to determine how best to translate the clinical trial efficacy of insulin into clinical practice effectiveness.

We observed that the proportions of patients who intensify with a third ADD have reduced only moderately during the last decade, despite the increasing availability of newer agents. Lipska and colleagues (2017) reported that overall glycaemic control in the USA did not change from $2006-2013^{29}$.

Advantage of this study is the availability of data from patients' medication lists that include prescribed medications within the EMR network and also medications that could be prescribed outside of the EMR. Furthermore, the CEMR database tracks longitudinal treatment adjustments, and contains comprehensive clinical information, which is usually not available in claims databases. We also have applied advanced data mining and statistical methods. Given unequal probabilities of receiving particular second-line agents in the realworld scenario, we have modelled treatment assignment with multinomial propensity scores, and then assessed adjusted outcomes of the study.

The limitations of this study include the non-availability of data on: (1) adherence and sideeffects; (2) diet and exercise; (3) socio-economic status; and (4) insurance type. Edelman and 
Polonsky (2017) highlighted alarmingly low rates of medication adherence as the main cause of the disconnect between results of real-world studies and clinical trials ${ }^{30}$. Importantly, this study focused only on those who continued the second-line therapy for a minimum of 6 months. Montvida et al (2017) recently reported higher discontinuation rates of incretins, compared to older treatment alternatives ${ }^{4}$.

To conclude, incretin-based therapies and TZDs offer a higher probability of long-term glycaemic achievements and their sustainability, comparing to SU and INS for metformintreated patients with type 2 diabetes. While the results of a large randomised control trial (GRADE) comparing glycaemic efficacy of major second-line therapies are not expected before 2020, our study provides the much-needed information to patients and clinicians in terms of the probability of sustainable glycaemic control with different therapy options ${ }^{31}$. 


\title{
FUNDING SOURCE
}

No separate funding was obtained for this study.

\begin{abstract}
APPROVAL
Research involved existing data, where the subjects could not be identified directly or through identifiers linked to the subjects. Thus, according to the US Department of Health and Human Services Exemption 4 (CFR 46.101(b)(4)), this study is exempt from ethics approval from an institutional review board and informed consent.
\end{abstract}

\section{ACKNOWLEDGEMENTS}

$\mathrm{OM}$ and SKP were responsible for the primary design of the study. OM conducted the data extraction. OM and SKP jointly conducted the statistical analyses. The first draft of the manuscript was developed by OM and SKP, and all authors contributed to the finalization of the manuscript. SKP had full access to all the data in the study and takes responsibility for the integrity of the data and the accuracy of the data analysis.

Melbourne EpiCentre gratefully acknowledges the support from the Australian Government Department of Education's National Collaborative Research Infrastructure Strategy (NCRIS) initiative through Therapeutic Innovation Australia. OM acknowledges the Ph. D. scholarship from Queensland University of Technology, Australia, and her co-supervisors Prof. Ross Young and Prof. Louise Hafner of the same University. No separate funding was obtained for this study. 


\section{DECLARATION OF INTERESTS}

SKP has acted as a consultant and/or speaker for Novartis, GI Dynamics, Roche, AstraZeneca, Guangzhou Zhongyi Pharmaceutical and Amylin Pharmaceuticals LLC. He has received grants in support of investigator and investigator initiated clinical studies from Merck, Novo Nordisk, AstraZeneca, Hospira, Amylin Pharmaceuticals, Sanofi-Avensis and Pfizer. OM has no conflict of interest to declare. JES has received honoraria or grant support from Merck Sharp and Dohme, Novo Nordisk, Eli Lilly, AstraZeneca, Sanofi-Aventis, Mylan Pharmaceuticals and Boehringer Ingelheim. 


\section{REFERENCES}

[1] American Diabetes Association. Standards of Medical Care in Diabetes-2017: Summary of Revisions. Diabetes Care. 2017; 40: S4-S5

[2] Turner RC, Cull CA, Frighi V, Holman RR, Group UPDS. Glycemic control with diet, sulfonylurea, metformin, or insulin in patients with type 2 diabetes mellitus: progressive requirement for multiple therapies (UKPDS 49). Jama. 1999; 281: 2005-2012

[3] Garber AJ, Abrahamson MJ, Barzilay Jl, et al. Consensus statement by the American association of clinical endocrinologists and American College of Endocrinology on the comprehensive type 2 diabetes management algorithm-2017 executive summary. Endocrine Practice. 2017; 23: 207-238

[4] Montvida O, Shaw J, Atherton JJ, Stringer F, Paul SK. Long-term trends in antidiabetes drug usage in the US: Real-world evidence in patients newly diagnosed with type 2 diabetes. Diabetes care. 2017: dc171414

[5] Giugliano D, Maiorino MI, Bellastella G, Esposito K. Comment on Edelman and Polonsky. Type 2 Diabetes in the Real World: The Elusive Nature of Glycemic Control. Diabetes Care 2017;40:1425-1432. Diabetes Care. 2018; 41: e17

[6] McCarthy MI. Painting a new picture of personalised medicine for diabetes. Diabetologia. 2017; 60: 793-799

[7] Rathmann W, Bongaerts B, Kostev K. Change in glycated haemoglobin levels after initiating second - line therapy in type 2 diabetes: a primary care database study. Diabetes, Obesity and Metabolism. 2016; 18: 840-843

[8] Thomsen RW, Baggesen LM, Søgaard M, et al. Early glycaemic control in metformin users receiving their first add-on therapy: a population-based study of 4,734 people with type 2 diabetes. Diabetologia. 2015; 58: 2247-2253

[9] Palmer SC, Mavridis D, Nicolucci A, et al. Comparison of clinical outcomes and adverse events associated with glucose-lowering drugs in patients with type 2 diabetes: a meta-analysis. Jama. 2016; 316: 313-324

[10] Maruthur NM, Tseng E, Hutfless S, et al. Diabetes Medications as Monotherapy or Metformin-Based Combination Therapy for Type 2 DiabetesA Systematic Review and MetaanalysisDiabetes Medications as Monotherapy or Metformin-Based Combination Therapy. Annals of internal medicine. 2016; 164: 740-751

[11] Bennett WL, Maruthur NM, Singh S, et al. Comparative effectiveness and safety of medications for type 2 diabetes: an update including new drugs and 2-drug combinations. Annals of internal medicine. 2011; 154: 602-613

[12] Zhang Y, McCoy RG, Mason JE, Smith SA, Shah ND, Denton BT. Second-line agents for glycemic control for type 2 diabetes: are newer agents better? Diabetes Care. 2014; 37: 1338-1345

[13] Centers for Disease Control and Prevention. National Diabetes Statistics Report: Estimates of Diabetes and Its Burden in the United States. Atlanta, GA: US Department of Health and Human Services. 2014:

[14] Crawford AG, Cote C, Couto J, et al. Comparison of GE Centricity Electronic Medical Record database and National Ambulatory Medical Care Survey findings on the prevalence of major conditions in the United States. Population health management. 2010; 13: 139-150 
[15] Brixner D, Said Q, Kirkness C, Oberg B, Ben-Joseph R, Oderda G. Assessment of cardiometabolic risk factors in a national primary care electronic health record database. Value in health. 2007; 10: S29-S36

[16] Paul SK, Shaw J, Montvida O, Klein K. Weight gain in insulin treated patients by BMI categories at treatment initiation: New evidence from real - world data in patients with type 2 diabetes. Diabetes, Obesity and Metabolism. 2016:

[17] Montvida O, Arandjelović O, Reiner E, Paul SK. Data Mining Approach to Estimate the Duration of Drug Therapy from Longitudinal Electronic Medical Records. Open Bioinformatics Journal. 2017; 10: 1-15

[18] Thomas G, Klein K, Paul S. Statistical challenges in analysing large longitudinal patient-level data: the danger of misleading clinical inferences with imputed data. J Indian Soc Agric Stat. 2014; 68: $39-54$

[19] Quan H, Sundararajan V, Halfon P, et al. Coding algorithms for defining comorbidities in ICD9-CM and ICD-10 administrative data. Medical care. 2005: 1130-1139

[20] Ridgeway G, McCaffrey DF, Morral AR, Burgette LF, Griffin BA. Toolkit for weighting and analysis of nonequivalent groups. 2014:

[21] Lunceford JK, Davidian M. Stratification and weighting via the propensity score in estimation of causal treatment effects: a comparative study. Statistics in medicine. 2004; 23: 2937-2960

[22] McCaffrey DF, Ridgeway G, Morral AR. Propensity score estimation with boosted regression for evaluating causal effects in observational studies. Psychological methods. 2004; 9: 403

[23] Woodcock J, Sharfstein JM, Hamburg M. Regulatory action on rosiglitazone by the US Food and Drug Administration. New England Journal of Medicine. 2010; 363: 1489-1491

[24] Tanne JH. FDA places" black box" warning on antidiabetes drugs. BMJ: British Medical Journal. 2007; 334: 1237

[25] Nissen SE, Wolski K. Effect of rosiglitazone on the risk of myocardial infarction and death from cardiovascular causes. New England Journal of Medicine. 2007; 356: 2457-2471

[26] Mamza J, Mehta R, Donnelly R, Idris I. Important differences in the durability of glycaemic response among second-line treatment options when added to metformin in type 2 diabetes: a retrospective cohort study. Annals of medicine. 2016; 48: 224-234

[27] Vaccaro O, Masulli M, Nicolucci A, et al. Effects on the incidence of cardiovascular events of the addition of pioglitazone versus sulfonylureas in patients with type 2 diabetes inadequately controlled with metformin (TOSCA. IT): a randomised, multicentre trial. The Lancet Diabetes \& Endocrinology. 2017:

[28] Edelman SV, Polonsky WH. Type 2 Diabetes in the Real World: The Elusive Nature of Glycemic Control. Diabetes Care. 2017; 40: 1425-1432

[29] Lipska KJ, Yao X, Herrin J, et al. Trends in drug utilization, glycemic control, and rates of severe hypoglycemia, 2006-2013. Diabetes care. 2017; 40: 468-475

[30] Carls GS, Tuttle E, Tan R-D, et al. Understanding the Gap Between Efficacy in Randomized Controlled Trials and Effectiveness in Real-World Use of GLP-1 RA and DPP-4 Therapies in Patients With Type 2 Diabetes. Diabetes Care. 2017; 40: 1469-1478

[31] Nathan DM, Buse JB, Kahn SE, et al. Rationale and design of the glycemia reduction approaches in diabetes: a comparative effectiveness study (GRADE). Diabetes Care. 2013; 36: 22542261 
Table 1: Characteristics at initiation of second-line anti-diabetic drug.

\begin{tabular}{|c|c|c|c|c|c|c|}
\hline & MET+SU & MET+DPP-4i & MET+GLP1RA & MET+INS & MET+TZD & ALL \\
\hline $\mathbf{N}$ & 90,572 & 29,308 & 6,696 & 21,827 & 14,678 & 163,081 \\
\hline Age (years) & $59(12)$ & $57(12)$ & $53(11)$ & $56(13)$ & $57(11)$ & $57(12)$ \\
\hline Male $^{\dagger}$ & $46,005(51)$ & $14,330(49)$ & $2,354(35)$ & $9,858(45)$ & $7,782(53)$ & $80,329(49)$ \\
\hline White $^{\dagger}$ & $63,338(70)$ & $20,366(69)$ & $5,100(76)$ & $14,267(65)$ & $10,256(70)$ & $113,327(69)$ \\
\hline Black $^{\dagger}$ & $11,703(13)$ & $3,618(12)$ & $616(9)$ & $3,690(17)$ & $1,434(10)$ & $21,061(13)$ \\
\hline Time from MET to 2nd drug (months) & $8.9(16.8)$ & $12.8(19.3)$ & $12.1(18.3)$ & $5.8(14.2)$ & $4.8(11.7)$ & $9.0(16.8)$ \\
\hline Follow-up from baseline (years) ${ }^{t}$ & $4.03(2.49)$ & $3.22(1.95)$ & $3.66(2.39)$ & $3.46(2.24)$ & $5.57(2.80)$ & $3.93(2.47)$ \\
\hline Therapy duration from baseline (months) & $38.3(26.3)$ & $29.6(20.1)$ & $28.4(21.0)$ & $37.9(26.0)$ & $35.8(26.1)$ & $36.0(25.3)$ \\
\hline Therapy duration e 12 months ${ }^{\dagger}$ & $77,779(86)$ & $23,327(80)$ & $5,061(76)$ & $18,729(86)$ & $12,040(82)$ & $136,936(84)$ \\
\hline Therapy duration e 24 months ${ }^{\dagger}$ & $56,324(62)$ & $14,746(50)$ & $3,090(46)$ & $13,472(62)$ & $8,297(57)$ & $95,929(59)$ \\
\hline HbA1c $(\%)^{\ddagger}$ & $8.4(1.8)$ & $8.2(1.7)$ & $7.8(1.6)$ & $9.3(2.3)$ & $7.9(1.7)$ & $8.4(1.9)$ \\
\hline \multicolumn{7}{|l|}{ HbA1c category $^{\dagger}$} \\
\hline $7-7.9 \%$ & $26,493(29)$ & $10,112(35)$ & $1,953(29)$ & $4,034(18)$ & $4,139(28)$ & $46,731(29)$ \\
\hline $8-9 \%$ & $18,701(21)$ & $5,726(20)$ & $1,027(15)$ & $3,838(18)$ & $2,295(16)$ & $31,587(19)$ \\
\hline $9.1-12 \%$ & $20,148(22)$ & $5,373(18)$ & $989(15)$ & $7,432(34)$ & $2,183(15)$ & $36,125(22)$ \\
\hline$>12 \%$ & $4,695(5)$ & $1,227(4)$ & $166(2)$ & $2,798(13)$ & $504(3)$ & $9,390(6)$ \\
\hline Weight (kg) & $98.3(24.5)$ & $98.9(24.2)$ & $109.5(25.9)$ & $99.8(26.2)$ & $100.2(23.9)$ & $99.3(24.8)$ \\
\hline BMI $\left(\mathrm{kg} / \mathrm{m}^{2}\right)^{t}$ & $34.5(7.7)$ & $34.5(7.6)$ & $38.5(8.1)$ & $35.2(8.4)$ & $34.8(7.6)$ & $34.8(7.8)$ \\
\hline \multicolumn{7}{|l|}{ BMI category $^{\dagger}$} \\
\hline Normal & $5,803(7)$ & $1,841(6)$ & $89(1)$ & $1,712(8)$ & $827(6)$ & $10,272(6)$ \\
\hline Overweight & $20,477(23)$ & $6,567(23)$ & $669(10)$ & $4,217(20)$ & $3,130(22)$ & $35,060(22)$ \\
\hline Grade 1 & $25,568(29)$ & $8,570(30)$ & $1,661(25)$ & $5,697(27)$ & $4,029(29)$ & $45,525(29)$ \\
\hline Grade 2+ & $35,853(41)$ & $11,788(41)$ & $4,144(63)$ & $9,587(45)$ & $6,012(43)$ & $67,384(43)$ \\
\hline
\end{tabular}

This article is protected by copyright. All rights reserved. 


\begin{tabular}{|c|c|c|c|c|c|c|}
\hline SBP $(\mathrm{mmHg})^{+}$ & $131(15)$ & $129(13)$ & $128(13)$ & $130(15)$ & $130(14)$ & $130(14)$ \\
\hline SBP e $140 \mathrm{mmHg}^{\dagger}$ & $22,164(25)$ & $5,807(20)$ & $1,084(17)$ & $5,022(23)$ & $3,088(22)$ & $37,165(23)$ \\
\hline LDL $(\mathrm{mg} / \mathrm{dL})^{\dagger}$ & $98(34)$ & $98(35)$ & $95(34)$ & $99(37)$ & $97(34)$ & $98(35)$ \\
\hline HDL $(\mathrm{mg} / \mathrm{dL})^{+}$ & $43(12)$ & $44(12)$ & $44(12)$ & $43(13)$ & $45(12)$ & $43(12)$ \\
\hline Triglycerides $(\mathrm{mg} / \mathrm{dL})^{\S}$ & $150(109,199)$ & $147(108,196)$ & $150(109,200)$ & $144(103,197)$ & $139(101,190)$ & $147(107,198)$ \\
\hline CVD, CKD, or Cancer ${ }^{\dagger}$ & $23,281(26)$ & $7,223(25)$ & $1,205(18)$ & $5,870(27)$ & $2,982(20)$ & $40,561(25)$ \\
\hline Cardiovascular disease $^{\dagger}$ & $18,031(20)$ & $5,406(18)$ & $852(13)$ & $4,675(21)$ & $2,276(16)$ & $31,240(19)$ \\
\hline Chronic kidney disease $^{\dagger}$ & $3,750(4)$ & $1,205(4)$ & $151(2)$ & $811(4)$ & $431(3)$ & $6,348(4)$ \\
\hline Cancer $^{\dagger}$ & $4,469(5)$ & $1,628(6)$ & $285(4)$ & $1,103(5)$ & $552(4)$ & $8,037(5)$ \\
\hline Neuropathy $^{\dagger}$ & $7,153(8)$ & $2,080(7)$ & $519(8)$ & $2,305(11)$ & $879(6)$ & $12,936(8)$ \\
\hline Retinopathy $^{\dagger}$ & $1,329(1)$ & $288(1)$ & $76(1)$ & $535(2)$ & $166(1)$ & $2,394(1)$ \\
\hline Depression $^{\dagger}$ & $14,925(16)$ & $5,427(19)$ & $1,576(24)$ & $4,200(19)$ & $2,145(15)$ & $28,273(17)$ \\
\hline Charlson Comorbidity Index & $1.7(1.1)$ & $1.7(1.1)$ & $1.6(0.9)$ & $1.8(1.2)$ & $1.5(0.9)$ & $1.7(1.1)$ \\
\hline
\end{tabular}

${ }^{\dagger} \mathrm{n}(\%) ;$ mean $(\mathrm{sd}) ;{ }^{\S}$ median $(\mathrm{IQR})$

BMI category - Normal: $<25 \mathrm{~kg} / \mathrm{m}^{2}$; Overweight: e 25 and $<30 \mathrm{~kg} / \mathrm{m}^{2}$; Grade 1: e 30 and $<35 \mathrm{~kg} / \mathrm{m}^{2}$; Grade 2+: e $35 \mathrm{~kg} / \mathrm{m}^{2}$; 
Table 2: Third-line anti-diabetic drug usage in the study cohort and two sub-cohorts ${ }^{\dagger}$.

\begin{tabular}{|c|c|c|c|c|c|c|c|}
\hline & & MET+SU & MET+DPP-4i & MET+GLP-1RA & MET+INS & MET+TZD & ALL \\
\hline Study Cohort & $\mathrm{N}$ & 90,572 & 29,308 & 6,696 & 21,827 & 14,678 & 163,081 \\
\hline Initiated third drug & $\mathrm{n}(\%$ from $\mathrm{N})$ & $49,255(54)$ & $15,248(52)$ & $3,513(52)$ & $7,275(33)$ & $10,006(68)$ & $85,297(52)$ \\
\hline $\begin{array}{l}\text { Time from 2nd to third drug, } \\
\text { months }\end{array}$ & mean $(\mathrm{sd})$ & $14.3(19.5)$ & $14.3(16.0)$ & $13.2(17.8)$ & $17.7(19.3)$ & $18.4(23.1)$ & $15.0(19.4)$ \\
\hline Added third drug within 6 months & $\mathrm{n} 1(\%$ from $\mathrm{N})$ & $24,600(27)$ & $6,053(21)$ & $1,725(26)$ & $2,627(12)$ & $4,260(29)$ & $39,265(24)$ \\
\hline - $\quad$ Most Popular third drug & $\begin{array}{l}\text { name; } \mathrm{n}(\% \text { from } \\
\mathrm{n} 1)\end{array}$ & TZD; 8,107 (33) & INS; $2,200(36)$ & INS; $1,189(69)$ & SU; 888 (34) & INS; $1,352(32)$ & $\begin{array}{l}\text { INS; } 11,054 \\
(28)\end{array}$ \\
\hline Second Popular third drug & $\begin{array}{l}\text { name; } \mathrm{n}(\% \text { from } \\
\mathrm{n} 1)\end{array}$ & \begin{tabular}{|l|} 
DPP-4I; 7,455 \\
$(30)$
\end{tabular} & SU; 2,073 (34) & SU; $193(11)$ & \begin{tabular}{|l|}
$\begin{array}{l}\text { DPP-4iI; } 703 \\
(27)\end{array}$ \\
\end{tabular} & \begin{tabular}{|l} 
DPP-4i; 1,236 \\
$(29)$
\end{tabular} & $\begin{array}{l}\text { DPP-4i; } \\
9,499(24)\end{array}$ \\
\hline Sub-cohort 1 & $\mathrm{~N} 2$ & 77,779 & 23,327 & 5,061 & 18,729 & 12,040 & 136,936 \\
\hline Added third drug within 6 months & $\mathrm{n}(\%$ from $\mathrm{N} 2)$ & $20,990(27)$ & $4,581(20)$ & $1,300(26)$ & $2,220(12)$ & $3,450(29)$ & $32,541(24)$ \\
\hline $\begin{array}{l}\text { Added third drug within 6-12 } \\
\text { months }\end{array}$ & $\mathrm{n} 2(\%$ from N2) & $4,265(5)$ & $1,860(8)$ & $293(6)$ & $1,076(6)$ & $682(6)$ & $8,176(6)$ \\
\hline - Most Popular third drug & $\begin{array}{l}\text { name; } \mathrm{n}(\% \text { from } \\
\mathrm{n} 2)\end{array}$ & $\begin{array}{l}\text { DPP-4I; } 1,737 \\
(41)\end{array}$ & SU; 975 (52) & SU; $104(35)$ & SU; $336(31)$ & SU; $340(50)$ & $\begin{array}{l}\text { DPP-4I; } \\
2,217(27)\end{array}$ \\
\hline - Second Popular third drug & $\begin{array}{l}\text { name; } \mathrm{n}(\% \text { from } \\
\mathrm{n} 2)\end{array}$ & INS; $1,074(25)$ & INS; 267 (14) & INS; $62(21)$ & $\begin{array}{l}\text { GLP1RA; } 269 \\
(25)\end{array}$ & $\begin{array}{l}\text { DPP-4I; } 160 \\
(23)\end{array}$ & $\begin{array}{l}\text { SU; } 1,755 \\
(21)\end{array}$ \\
\hline Sub-cohort2 & N3 & 56,324 & 14,746 & 3,090 & 13,472 & 8,297 & 95.929 \\
\hline Added third drug within 6 months & $\mathrm{n}(\%$ from $\mathrm{N} 3)$ & $15,074(27)$ & $2,549(17)$ & $800(26)$ & $1,521(11)$ & $2,309(28)$ & $22,253(23)$ \\
\hline $\begin{array}{l}\text { Added third drug within 6-12 } \\
\text { months }\end{array}$ & $\mathrm{n}(\%$ from $\mathrm{N} 3)$ & $2,867(5)$ & $1,124(8)$ & $168(5)$ & $756(6)$ & $471(6)$ & $5,386(6)$ \\
\hline $\begin{array}{l}\text { Added third drug within 12-24 } \\
\text { months }\end{array}$ & $\mathrm{n} 3(\%$ from N3) & $5,302(9)$ & $1,833(12)$ & $319(10)$ & $1,070(8)$ & $645(8)$ & $9,169(10)$ \\
\hline - $\quad$ Most Popular third drug & $\begin{array}{l}\text { name; } n(\% \text { from } \\
\text { n3) }\end{array}$ & $\begin{array}{l}\text { DPP-4I; } 2,356 \\
(44)\end{array}$ & SU; 959 (52) & SU; $113(35)$ & SU; $301(28)$ & SU; 297 (46) & $\begin{array}{l}\text { DPP-4I; } \\
2,876(31)\end{array}$ \\
\hline
\end{tabular}

This article is protected by copyright. All rights reserved. 


\begin{tabular}{|l|l|l|l|l|l|l|}
\hline- Second Popular third drug & $\begin{array}{l}\text { name; } \mathrm{n}(\% \text { from } \\
\mathrm{n} 3)\end{array}$ & INS; 1,225 (23) & $\begin{array}{l}\text { SGLT2; 274 } \\
(15)\end{array}$ & INS; 63 (20) & $\begin{array}{l}\text { DPP-4I; 269 } \\
(25)\end{array}$ & $\begin{array}{l}\text { DPP-4I; 201 } \\
(31)\end{array}$ \\
\hline
\end{tabular}

MET: metformin; SU: sulfonylurea; DPP-4i: dipeptidyl peptidase-4 inhibitor; GLP-1RA: glucagon-like peptide-1 receptor agonist; INS: insulin; TZD: thiazolidinedione;

${ }^{\dagger}$ Duration of second-line agent e 6 months/ e 12 months/ e 24 months in the study cohort/ sub-cohort 1/ sub-cohort 2, respectively. 
Figure 1: Among patients who had at least 2 years of follow-up in the study cohort, the proportion (95\% CI) of patients who initiated third ADD within 2 years of second ADD,.
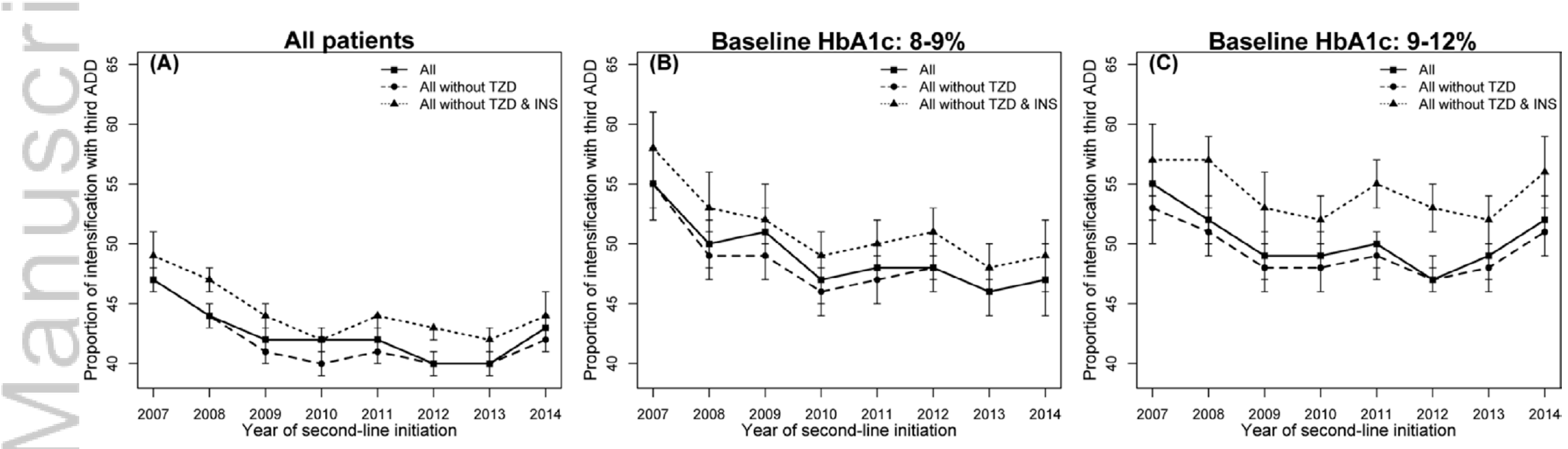
Figure 2: At 6, 12, and 24 months of second-line initiation, adjusted probability (95\% CI) to (A) reduce HbA1c below 7\% without adding third ADD, from baseline HbA1c of 7.5-7.9\%; (B) to sustain 6 month achievement without adding third ADD; (C) reduce HbA1c below 7.5\% without adding third ADD, from baseline $\mathrm{HbA1c}$ of $8-9 \%$; (D) to sustain 6 month achievement without adding third ADD; (E) reduce HbA1c below 7.5\% (irrespective of third ADD), from baseline HbAlc of 9.1-12\%; (F) to sustain 6 month achievement (irrespective of third ADD).
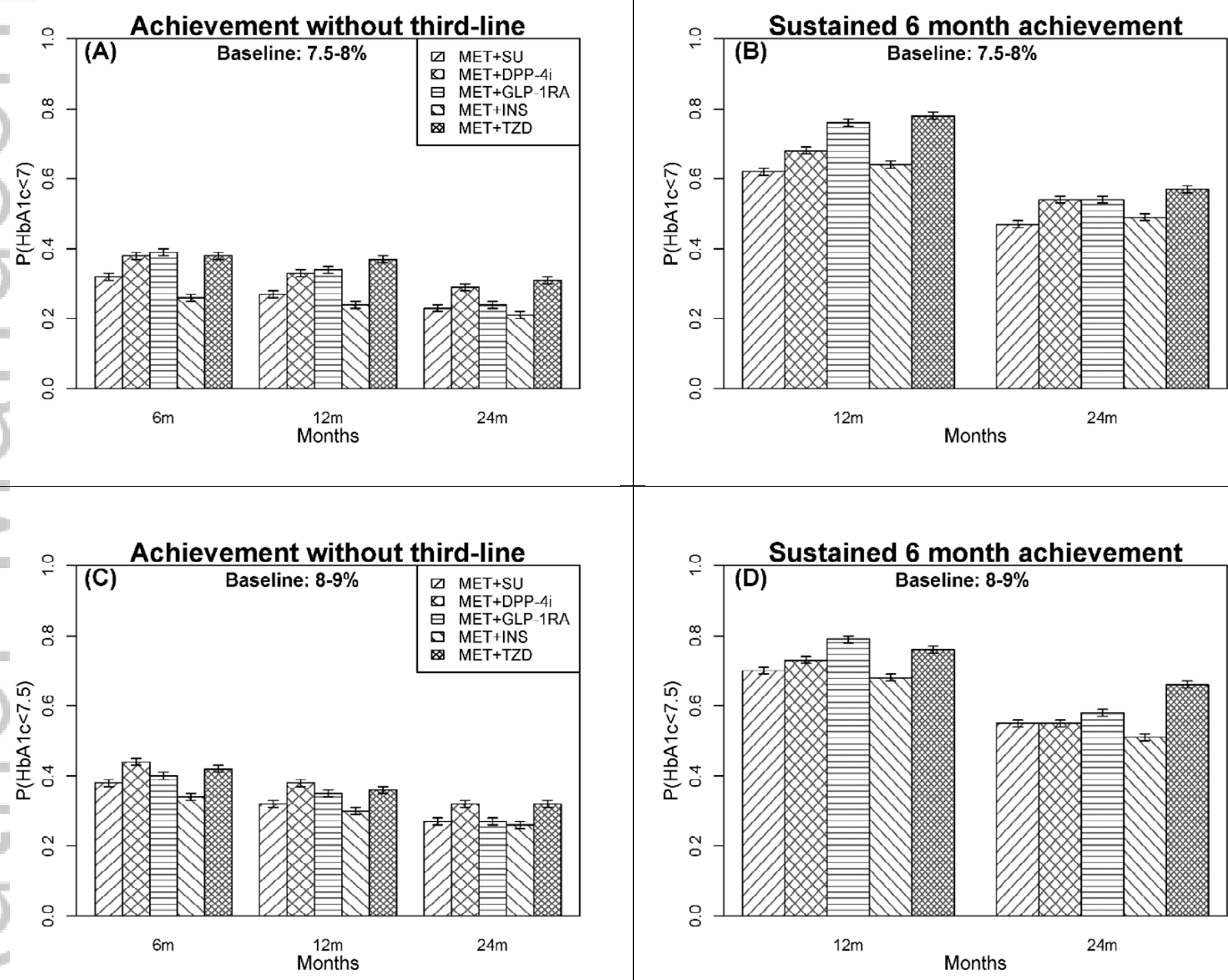

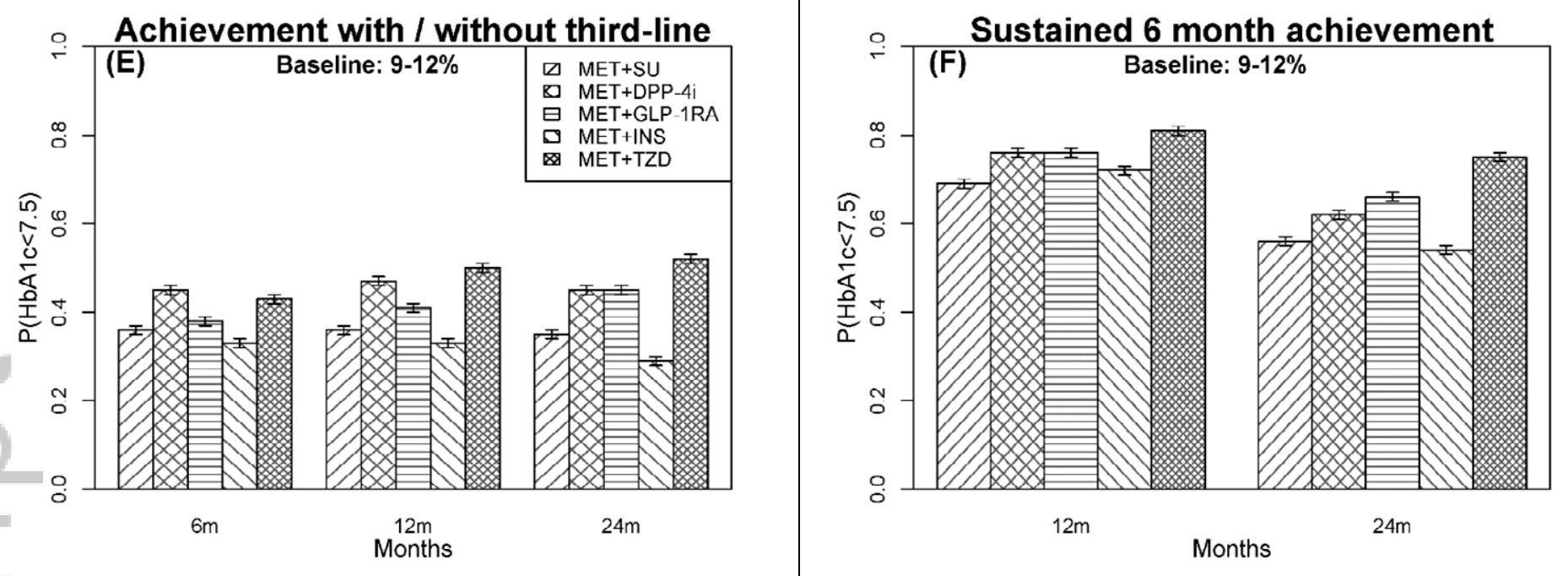

This article is protected by copyright. All rights reserved. 


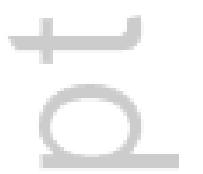

This article is protected by copyright. All rights reserved. 


\section{University Library}

\section{- M M N E R VA A gateway to Melbourne's research publications}

Minerva Access is the Institutional Repository of The University of Melbourne

Author/s:

Montvida, O;Shaw, JE;Blonde, L;Paul, SK

Title:

Long-term sustainability of glycaemic achievements with second-line antidiabetic therapies in patients with type 2 diabetes: A real-world study

Date:

2018-07-01

Citation:

Montvida, O., Shaw, J. E., Blonde, L. \& Paul, S. K. (2018). Long-term sustainability of glycaemic achievements with second-line antidiabetic therapies in patients with type 2 diabetes: A real-world study. DIABETES OBESITY \& METABOLISM, 20 (7), pp.1722-1731. https://doi.org/10.1111/dom.13288.

Persistent Link:

http://hdl.handle.net/11343/283734 